\title{
Interconnection-Based Performance Analysis for a Class of Decentralized Controllers
}

\author{
Somayeh Sojoudi ${ }^{a}$, Amir G. Aghdam ${ }^{\text {b }}$ \\ ${ }^{a}$ Department of Control and Dynamical Systems, California Institute of Technology, Pasadena, CA 91125, USA \\ ${ }^{\mathrm{b}}$ Department of Electrical and Computer Engineering, Concordia University, Montréal, QC H3G 1M8, Canada
}

\begin{abstract}
This paper is concerned with decentralized controller design for large-scale interconnected systems of pseudo-hierarchical structure. Given such a system, one can use existing techniques to design a decentralized controller for the reference hierarchical model, obtained by eliminating certain weak interconnections of the original system. Although this indirect controller design is appealing as far as the computational complexity is concerned, it does not necessarily result in satisfactory performance for the original pseudo-hierarchical system. A LQ cost function is defined in order to evaluate the performance discrepancy between the pseudo-hierarchical system and its reference hierarchical model under the designed decentralized controller. A discrete Lyapunov equation is then solved to compute this performance index. However, due to the large-scale nature of the system, this equation cannot be handled efficiently in many real-world systems. Thus, attaining an upper bound on this cost function can be more desirable than finding its exact value, in practice. For this purpose, a novel technique is proposed which only requires solving a simple LMI optimization problem with three variables. The problem is then reduced to a scalar optimization problem, for which an explicit solution is provided. It is also shown that when the original model is exactly hierarchical, then the upper bounds obtained from the LMI and scalar optimization problems will both be equal to zero.
\end{abstract}

Key words: Decentralized control, optimization, matrix inequality.

\section{Introduction}

Many real-world systems can be described by large-scale interconnected models (Antoulas et al., 2001). There is a great deal of interest in performance analysis and control synthesis of large-scale systems. A key practical consideration in designing a controller for this type of system is to rely on local information as much as possible. Decentralized control theory was introduced in the literature to address this consideration, and reduce the complexity of the control implementation for large-scale systems. Distinctive aspects of decentralized control systems have been well-documented in the last three decades (Siljak, 1991; Jamshidi, 1997). A decentralized controller consists of a number of isolated local controllers corresponding to the subsystems of the large-scale system. For the sake of simplicity of the control design problem, it is often desirable that the large-scale system possesses a hierarchical structure (Stankovic et al., 1989; Lavaei et al.,

\footnotetext{
* This work has been supported by the Natural Sciences and Engineering Research Council of Canada (NSERC).

Email addresses: sojoudi@cds.caltech.edu (Somayeh Sojoudi), aghdam@ece.concordia.ca (Amir G. Aghdam).
}

2008). Note that a hierarchical model refers to an interconnected system whose subsystems can be renumbered in such a way that the corresponding transfer function matrix becomes lower block-triangular. The control design problem for a hierarchical system can be broken down into a number of parallel design subproblems corresponding to different subsystems. The advantage of such design techniques is twofold: the control design procedure is far simpler for a number of low-order subsystems compared to that for one high-order system, and at the same time parallel computation is very fast.

Many important physical cooperative control applications with a leader-follower configuration such as formation flight, underwater vehicles, automated highway systems, satellite constellation, etc. have a hierarchical structure (Tanner et al., 2004; Fax et al., 2004; Stilwell et al., 2000). Furthermore, it is shown in Aghdam et al. (2006) that under certain conditions, a continuoustime non-hierarchical system can have a hierarchical discrete-time equivalent model (this model represents the continuous-time system only at the sampling time instants). It is straightforward to show that a set of stabilizing local controllers obtained by neglecting all 
the interconnections between the subsystems constitute a stabilizing decentralized controller for the original hierarchical system. This is quite beneficial in the sense that it provides a simple control design method for hierarchical systems, as far as stability is concerned. In addition, a technique is given in Lavaei et al. (2006) to design a near-optimal decentralized controller for hierarchical systems. This idea is further developed in Lavaei et al. (2007) to decentralize any given centralized controller while its fundamental properties are preserved. Although decentralized control design for hierarchical systems has been extensively investigated in the past several years, there are only a few control design techniques for general large-scale systems, due to the complexity of the problem. Furthermore, there is no efficient performance evaluation method when the controller is designed for the system after some structural modifications.

On the other hand, there exist numerous non-hierarchical systems which are "close" to being hierarchical. Such systems have a few weak interconnections between their subsystems, whose elimination would result in an exact hierarchical structure. This type of systems will be referred to as pseudo-hierarchical systems throughout the paper, and the hierarchical model obtained by eliminating minimum number of "weak" interconnections in a pseudo-hierarchical system will be called the corresponding reference hierarchical model. Given a pseudo-hierarchical large-scale system, a decentralized controller can be obtained for the corresponding reference hierarchical model by designing each local controller separately using available techniques. Even though this straightforward approach is appealing as far as the computational complexity is concerned, the decentralized controller obtained will not necessarily meet the design specifications for the original pseudohierarchical system. In fact, it may not even stabilize the original system, although it stabilizes the corresponding reference hierarchical model. Apart from the stability issue (which is not a concern if the interconnections which are removed to obtain the reference hierarchical model are sufficiently weak), the performance of the pseudo-hierarchical system under this controller can be quite poor. Thus, it is important to carry out a proper performance analysis for the system in order to make certain that this indirect design technique is suitable for the given pseudo-hierarchical large-scale system.

This paper deals with the performance analysis for decentralized large-scale systems. It is assumed that a decentralized controller is provided for the reference hierarchical model of a pseudo-hierarchical system which meets certain control objectives. Moreover, it is supposed that the above-mentioned controller stabilizes the pseudo-hierarchical system, although closed-loop performance can be poor. A proper LQ cost function is defined to assess the discrepancy between the pseudohierarchical system and the corresponding reference hi- erarchical model under this decentralized controller. The smaller this performance index is, the closer the two closed-loop systems are to each other. Obtaining this cost function involves solving a discrete Lyapunov equation. However, due to the large-scale nature of the system, this equation might be difficult to handle, in general. Alternatively, it would be very useful to attain an upper bound on this cost function. A novel technique is proposed to address this objective, and it is subsequently shown that a LMI optimization problem with only three variables needs to be solved in order to compute this bound. This problem is also simplified and an explicit bound is proposed, without having to solve any optimization problem. One of the distinguishing features of this work is that it presents a simple technique for performance evaluation of pseudo-hierarchical decentralized systems. To elucidate that the obtained bounds are not too conservative in general, it is proved that if the difference between the pseudo-hierarchical system and the corresponding reference hierarchical model is sufficiently close to zero, so are these bounds. In particular, if the original model is exactly hierarchical, then both bounds will be equal to zero.

Another application of this work is in the control of overlapping systems, which has drawn much attention in recent years (Iftar, 1993; Stankovic et al., 2000; Siljak et al., 2005; Zecevic et al., 2005). Note that an overlapping system refers to a collection of subsystems, some of which share part of their states. For such a system, it is known that the most effective control structure is an overlapping one, in which any pair of local controllers can share data only if their corresponding subsystems share their states. The most prevalent method for designing an overlapping controller is to employ the inclusion principle to convert the problem to the conventional decentralized framework (Chu et al., 2005). This is achieved by expanding the system into another system whose interconnections are all weak. Hence, a set of local controllers (which will altogether be referred to as a decentralized controller) can be designed for the individual subsystems of the expanded system by neglecting the interconnections. The overall decentralized controller will eventually be contracted to attain a decentralized overlapping controller for the original system. Since all the interconnections in the expanded system are weak, and moreover they are disregarded in the controller design, a proper performance analysis for this design technique would be very useful. To this end, the expanded system can be considered as a pseudo-hierarchical model, and the expanded system with neglected interconnections can be regarded as the corresponding reference hierarchical model. Thus, the performance analysis for the expanded system will fit into the formulation considered here, as a special case.

The organization of this paper is as follows. In Section II, some preliminary results are provided and the problem is formulated. The main results are derived in Sec- 
tion III, which are illustrated by three numerical examples in Section IV. Finally, some concluding remarks are drawn in Section V.

\section{Preliminaries and problem formulation}

Consider a large-scale interconnected system $\mathcal{S}$ consisting of $\nu$ subsystems, where its $i$-th subsystem $S_{i}$ is represented by:

$$
\begin{aligned}
& x_{i}[k+1]=\sum_{j=1}^{\nu} A_{i j} x_{j}[k]+B_{i} u_{i}[k] \\
& y_{i}[k]=C_{i} x_{i}[k], \quad i \in \bar{\nu}:=\{1,2, \cdots, \nu\}
\end{aligned}
$$

In the above equation, $x_{i}[k] \in \mathbb{R}^{n_{i}}, u_{i}[k] \in \mathbb{R}^{m_{i}}$ and $y_{i}[k] \in \mathbb{R}^{r_{i}}$ stand for the state, input and output of $S_{i}$, respectively. Sketch now a digraph $\mathcal{G}$ associated with the system $\mathcal{S}$ as follows:

- Assign $\nu$ vertices, one for each subsystem of $\mathcal{S}$.

- For any $i, j \in \bar{\nu}, i \neq j$, connect vertex $i$ to vertex $j$ with a directed edge if $A_{i j} \neq 0$.

- For any $i, j \in \bar{\nu}$, if there is an edge between vertex $i$ and vertex $j$, attribute the weight $\left\|A_{i j}\right\|_{F}$ to that edge, where $\|\cdot\|_{F}$ denotes the Frobenius norm operator.

The graph $\mathcal{G}$ specifies the topology of information transfer between the subsystems. From this perspective, it plays an important role in the stability and stabilizability analysis of the system. If the graph $\mathcal{G}$ has no directed cycles, then the system $\mathcal{S}$ is said to be acyclic or hierarchical (Lavaei et al., 2006). For any $i \in \bar{\nu}$, define the isolated subsystem $\bar{S}_{i}$ as:

$$
\begin{aligned}
& \bar{x}_{i}[k+1]=A_{i i} \bar{x}_{i}[k]+B_{i} \bar{u}_{i}[k] \\
& \bar{y}_{i}[k]=C_{i} \bar{x}_{i}[k]
\end{aligned}
$$

For the case when the graph $\mathcal{G}$ is acyclic, a stabilizing decentralized controller can be obtained by designing $\nu$ local controllers separately such that the $i$-th local controller stabilizes the isolated subsystem $\bar{S}_{i}$, for all $i \in \bar{\nu}$. This simple fact implies that when the graph $\mathcal{G}$ is acyclic, the decentralized controller design can be quite straightforward (as far as the stability is concerned). As noted earlier, several methods are proposed in the literature to design a LTI decentralized controller for a hierarchical system in order to achieve any pre-specified objectives (Lavaei et al., 2006, 2008).

In the general case, when the graph $\mathcal{G}$ is not acyclic, one can remove certain edges of $\mathcal{G}$ to obtain an acyclic graph, and design the local controllers for the resultant system, as described before. However, the controller obtained may not perform satisfactorily when applied to the original system, if the interconnections neglected in the control design are not sufficiently weak. It is stated in
Krtolica et al. (1980) that there exist numerous systems for which all the edges of the graph $\mathcal{G}$ can be removed and designs local controllers accordingly. Nevertheless, in order to convert the graph $\mathcal{G}$ to an acyclic one, only a subset of the edges are required to be removed (such a subset is not necessarily unique).

Note that the $\varepsilon$-decomposition technique developed in the literature proposes a systematic method for eliminating certain interconnections of the system whose strength is below a specific level (see Sezer et al. (1986, 1991); Zecevic et al. (1994)). One can adopt above technique to identify the links whose elimination is not detrimental, and could lead to a hierarchical model.

Assume now that some of the edges are removed to obtain hierarchical model $\mathcal{S}_{h}$, and that a LTI decentralized controller $\mathcal{K}$ is designed for the resultant model using the existing methods. Once this controller is applied to the original system $\mathcal{S}$, the closed-loop system may perform poorly, and may even be unstable. Therefore, it is desired to evaluate the performance of the system $\mathcal{S}$ under the controller $\mathcal{K}$, with respect to its hierarchical counterpart (i.e. the hierarchical model under the same controller $\mathcal{K}$ ). To this end, it is assumed that the closed-loop system is stable, which is a requirement for performance degradation analysis in this work. It is worth mentioning that the closed-loop stability is guaranteed if the interconnections neglected in control design procedure are sufficiently weak.

In the sequel, the hierarchical model $\mathcal{S}_{h}$ under the designed LTI decentralized controller $\mathcal{K}$ is represented as:

$$
x_{h}[k+1]=A_{h} x_{h}[k]
$$

and the original system $\mathcal{S}$ under the same controller as:

$$
x_{c}[k+1]=A_{c} x_{c}[k]
$$

(it is important to note that both (3) and (4) are closedloop equations). Furthermore, set $x_{c}[0]=x_{h}[0]$. With no loss of generality, the matrix $A_{h}$ can be assumed to be lower-block triangular.

Remark 1 In the case when a decentralized overlapping controller is to be designed for an overlapping system by mean of the inclusion principle, the expanded system (obtained from the original overlapping system) under the decentralized controller designed after neglecting all the interconnections is expressed by (4). The expanded system with nullified interconnections under the abovementioned decentralized controller can then be described by (3), as a special case of a hierarchical model (this point is clarified in Example 1).

In order to assess the closeness of the systems given in (3) and (4), one can measure the discrepancy between 
the states $x_{h}[k]$ and $x_{c}[k]$. This can be evaluated by the following performance index:

$$
J_{d}=\sum_{k=0}^{\infty}\left(x_{c}[k]-x_{h}[k]\right)^{T}\left(x_{c}[k]-x_{h}[k]\right)
$$

Definition 1: Define the performance indices $J_{c}$ and $J_{h}$ as:

$$
J_{c}=\sum_{k=0}^{\infty} x_{c}[k]^{T} x_{c}[k], \quad J_{h}=\sum_{k=0}^{\infty} x_{h}[k]^{T} x_{h}[k]
$$

Definition 2: Given a positive real value $\mu$, the controller $\mathcal{K}$ is said to be $\mu$-suboptimal, if the inequality $\frac{J_{d}}{J_{h}}<\mu$ holds.

Some works such as Krtolica et al. (1980) define the degree of suboptimality based on the ratio $\frac{J_{c}}{J_{h}}$, as opposed to $\frac{J_{d}}{J_{h}}$. However, it is manifest that the smallness of $\frac{J_{c}}{J_{h}}$ does not necessarily imply the closeness of $x_{c}[k]$ and $x_{h}[k]$. The objective here is to obtain a proper and easyto-compute $\mu$ by which the controller $\mathcal{K}$ is suboptimal. The following practical restrictions are imposed.

Assumption 1: Given the system $\mathcal{S}$, a discretecannot be solved efficiently due to Lyapunov equation with the order of the system (i.e. $n=\sum_{i=1}^{\nu} n_{i}$ ) the large-scale nature of the system, whereas a discrete Lyapunov equation with the order of any associated subsystem $\mathcal{S}_{i}, i \in \bar{\nu}$, can be handled more efficiently.

Assumption 2: Although solving a Lyapunov equation of order $n$ is cumbersome, lower and upper bounds on the eigenvalues of a matrix of order $n$ may be obtained efficiently.

It is important to note in Assumption 2 that in general solving a Lyapunov equation of order $n$ is much more difficult than estimating the eigenvalues of a matrix of order $n$. In fact, the former problem involves $n^{2}$ variables while the latter one includes $n+1$ variables only (regardless of their linearity or bilinearity).

It is evident that $J_{h}$ in (6) satisfies the relation:

$$
J_{h}=x_{h}[0]^{T} P_{h} x_{h}[0]
$$

where:

$$
A_{h}^{T} P_{h} A_{h}-P_{h}+I=0
$$

In order to obtain the main results of the paper, one more assumption is required to be made.

Assumption 3: The closed-loop system given in (4) is stable with the Lyapunov matrix $P_{h}$.
It is to be noted that Assumption 3 is more restrictive than just the stability condition for the system (4), and is met when the removed edges have sufficiently small weights. Various sufficient conditions are provided in the literature to ensure the validity of this assumption.

\section{Main results}

In what follows, the performance deviation $J_{d}$ will be formulated.

Lemma 1 The performance index $J_{d}$ is equal to:

$$
\left[x_{h}[0]^{T} x_{h}[0]^{T}\right] P_{d}\left[\begin{array}{l}
x_{h}[0] \\
x_{h}[0]
\end{array}\right]
$$

where:

$$
\left[\begin{array}{cc}
A_{h} & 0 \\
0 & A_{c}
\end{array}\right] P_{d}\left[\begin{array}{cc}
A_{h} & 0 \\
0 & A_{c}
\end{array}\right]^{T}-P_{d}+\left[\begin{array}{cc}
I & -I \\
-I & I
\end{array}\right]=0
$$

Proof: Augmenting the closed-loop systems (3) and (4) results in:

$$
\left[\begin{array}{c}
x_{h}[k+1] \\
x_{c}[k+1]
\end{array}\right]=\left[\begin{array}{cc}
A_{h} & 0 \\
0 & A_{c}
\end{array}\right]\left[\begin{array}{l}
x_{h}[k] \\
x_{c}[k]
\end{array}\right]
$$

On the other hand, the performance index $J_{d}$ can be rewritten as:

$$
J_{d}=\sum_{k=0}^{\infty}\left[x_{h}[k]^{T} x_{c}[k]^{T}\right]\left[\begin{array}{cc}
I & -I \\
-I & I
\end{array}\right]\left[\begin{array}{l}
x_{h}[k] \\
x_{c}[k]
\end{array}\right]
$$

It is well-known that the above expression can be written as (9), where the matrix $P_{d}$ satisfies the equation (10). This completes the proof.

Due to Assumption 1, the performance deviation $J_{d}$ cannot be directly computed from Lemma 1 in order to compute the ratio $\frac{J_{d}}{J_{h}}$ precisely. Hence, the notion of $\mu$ optimality is used here in order to obtain a reasonable upper bound on this ratio, which is carried out in the sequel.

Lemma 2 Given a matrix $H$ of proper dimension, assume the following inequality is satisfied:

$$
\left[\begin{array}{cc}
A_{h} & 0 \\
0 & A_{c}
\end{array}\right]^{T} H\left[\begin{array}{cc}
A_{h} & 0 \\
0 & A_{c}
\end{array}\right]-H+\left[\begin{array}{cc}
I & -I \\
-I & I
\end{array}\right]<0
$$


Then, the inequality given below holds:

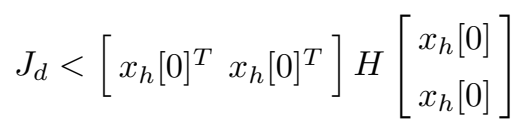

Proof: It can be concluded from the relations (10) and (13) that:

$$
\left[\begin{array}{cc}
A_{h} & 0 \\
0 & A_{c}
\end{array}\right]^{T}\left(H-P_{d}\right)\left[\begin{array}{cc}
A_{h} & 0 \\
0 & A_{c}
\end{array}\right]-\left(H-P_{d}\right)<0
$$

Since both of the matrices $A_{c}$ and $A_{h}$ are assumed to be Schur, it results from the above inequality that $P_{d}<H$. The proof follows immediately from this result and the equation (9).

Now, let the following optimization problem be introduced, which will be used later to show the $\mu$ suboptimality of the controller.

Problem 1: Find the infimum of the objective function $k_{1}+2 k_{2}+k_{3}$ for the variables $k_{1}, k_{2}$ and $k_{3}$, subject to:

$$
\left[\begin{array}{cr}
\left(1-k_{1}\right) I & k_{2}\left(A_{h}^{T} P_{h} A_{c}-P_{h}\right)-I \\
k_{2}\left(A_{c}^{T} P_{h} A_{h}-P_{h}\right)-I & k_{3}\left(A_{c}^{T} P_{h} A_{c}-P_{h}\right)+I
\end{array}\right]<0
$$

Remark 2 Problem 1 is a LMI optimization which can be efficiently handled using proper software tools such as YALMIP or SOSTOOLS (Lofberg, 2004; Prajna et al., 2004).

Theorem 1 The controller $\mathcal{K}$ is $\mu$-suboptimal, where $\mu$ denotes the infimum obtained by solving Problem 1.

Proof: Consider any real scalars $k_{1}, k_{2}$ and $k_{3}$ satisfying the inequality (16) given in Problem 1. Using the equation given in (8), this inequality can be rewritten as:

$$
\left[\begin{array}{cc}
A_{h} & 0 \\
0 & A_{c}
\end{array}\right]^{T} H\left[\begin{array}{cc}
A_{h} & 0 \\
0 & A_{c}
\end{array}\right]-H+\left[\begin{array}{cc}
I & -I \\
-I & I
\end{array}\right]<0
$$

if the matrix $H$ is chosen as:

$$
H=\left[\begin{array}{ll}
k_{1} P_{h} & k_{2} P_{h} \\
k_{2} P_{h} & k_{3} P_{h}
\end{array}\right]
$$

Therefore, it can be inferred from Lemma 2 that:

$$
\begin{aligned}
J_{d}< & {\left[x_{h}[0]^{T} x_{c}[0]^{T}\right] H\left[\begin{array}{l}
x_{h}[0] \\
x_{c}[0]
\end{array}\right]=} \\
& \left(k_{1}+2 k_{2}+k_{3}\right) x_{h}[0]^{T} P_{h} x_{h}[0]= \\
& \left(k_{1}+2 k_{2}+k_{3}\right) J_{h}
\end{aligned}
$$

(note that $x_{h}[0]=x_{c}[0]$ ). Thus:

$$
\frac{J_{d}}{J_{h}}<k_{1}+2 k_{2}+k_{3}
$$

The above inequality implies that the minimum of the objective function $k_{1}+2 k_{2}+k_{3}$ is to be minimized, and this completes the proof.

Theorem 1 states that the solution of Problem 1 provides an upper bound on the ratio $\frac{J_{d}}{J_{h}}$. It is interesting to note that the inequality constraint of this optimization problem is always feasible. To prove this, it suffices to choose $k_{1}=2, k_{2}=0$ and let $k_{3}$ be a very large number. Note from Assumption 3 that the matrix $A_{c}^{T} P_{h} A_{c}-P_{h}$ is negative definite; hence, the inequality (16) holds.

Due to Assumption 1 and the large-scale nature of the system $\mathcal{S}$, Problem 1 may not be easily solvable in practice. This is mainly because of the matrix constraint (16) which becomes sophisticated for large-scale systems. Thus, it is desirable to convert the matrix inequality (16) into a scalar form. This objective will be addressed in the sequel.

Problem 2: Find the infimum of the objective function $k_{1}+2 k_{2}+k_{3}$ for the variables $k_{1}, k_{2}$ and $k_{3}$ subject to the scalar inequalities $k_{1}>1$ and:

$$
\left(k_{1}-1\right)\left(-1+k_{3} m_{1}\right)-1-k_{2}^{2} m_{2}-k_{2} m_{3}>0
$$

where:

$$
\begin{aligned}
& m_{1}=\underline{\lambda}\left(-R_{2}\right), \quad m_{2}=\bar{\lambda}\left(R_{1} R_{1}^{T}\right), \\
& m_{3}=\bar{\lambda}\left(-R_{1}-R_{1}^{T}\right), \\
& R_{1}=A_{c}^{T} P_{h} A_{h}-P_{h}, \quad R_{2}=A_{c}^{T} P_{h} A_{c}-P_{h}
\end{aligned}
$$

(the notations $\bar{\lambda}(\cdot)$ and $\underline{\lambda}(\cdot)$ represent the maximum and minimum magnitudes of the eigenvalues of a matrix, respectively).

Theorem 2 Denote with $\mu$ the infimum obtained by solving Problem 2. Then, the controller $\mathcal{K}$ is $\mu$ suboptimal.

Proof: The inequality constraint of Problem 1 can be rearranged as: 


$$
\left[\begin{array}{cc}
\left(k_{3} R_{2}+I\right) & \left(k_{2} R_{1}-I\right) \\
\left(k_{2} R_{1}^{T}-I\right) & \left(1-k_{1}\right) I
\end{array}\right]<0
$$

Applying the Schur complement formula to the above inequality results in:

$$
\left(k_{1}-1\right)\left(k_{3} R_{2}+I\right)+\left(k_{2} R_{1}-I\right)\left(k_{2} R_{1}^{T}-I\right)<0
$$

It is easy to verify that the matrix inequality (24) is guaranteed to hold, provided the scalar inequality given below is satisfied:

$\underline{\lambda}\left(\left(k_{1}-1\right)\left(-k_{3} R_{2}-I\right)\right)>\bar{\lambda}\left(\left(I-k_{2} R_{1}\right)\left(I-k_{2} R_{1}^{T}\right)\right)$

Choose some scalars $k_{1}, k_{2}, k_{3}$ satisfying the inequality (21). It can be deduced from the above discussion and the result of Theorem 1 that in order to prove Theorem 2 it suffices to substantiate the validity of the inequality (25). To this end, one can use the following equation:

$$
\underline{\lambda}\left(\left(k_{1}-1\right)\left(-k_{3} R_{2}-I\right)\right)=\left(k_{1}-1\right)\left(-1+k_{3} m_{1}\right)
$$

Moreover, it results from Lemma 2.1 in Lee (2004) that:

$$
\begin{aligned}
& \bar{\lambda}\left(\left(I-k_{2} R_{1}\right)\left(I-k_{2} R_{1}^{T}\right)\right)= \\
& 1+\bar{\lambda}\left(k_{2}\left(-R_{1}-R_{1}^{T}\right)+k_{2}^{2} R_{1} R_{1}^{T}\right) \\
& \leq 1+k_{2} \bar{\lambda}\left(-R_{1}-R_{1}^{T}\right)+k_{2}^{2} \bar{\lambda}\left(R_{1} R_{1}^{T}\right) \\
& \leq 1+k_{2} m_{3}+k_{2}^{2} m_{2}
\end{aligned}
$$

The relations (21), (26) and (27) altogether lead to the inequality (25).

Remark 3 Similar to the previous case, it can be shown that the constraints of Problem 2 are always feasible (by considering $k_{1}=2, k_{2}=0$ and a sufficiently large number for $\left.k_{3}\right)$. It is to be noted that $m_{1}$ is positive by definition.

Remark 4 Since the statement of Problem 2 is obtained by reducing the matrix constraint in Problem 1 to some scalar constraints, the upper bound proposed for $\mu$ in Theorem 2 is more conservative than the one given in Theorem 1 .

To solve any of the two problems introduced in this paper, the Lyapunov matrix $P_{h}$ needs to be obtained first. As a consequence of Assumption 1, this matrix cannot be computed efficiently using the conventional methods. However, since the matrix $A_{h}$ (which is required for obtaining $P_{h}$ ) is assumed to be lower block-triangular, $P_{h}$ can be found by solving a number of Lyapunov and Sylvester equations of subsystems' orders (as opposed to the system's order), successively. This fact is elaborated in detail in the appendix.
Theorem 3 Denote the optimal values of the variables $k_{1}, k_{2}, k_{3}$ in Problem 2 with $k_{1}^{*}, k_{2}^{*}, k_{3}^{*}$. The triple $\left(k_{1}^{*}, k_{2}^{*}, k_{3}^{*}\right)$ satisfies either the set of equations:

$$
\begin{aligned}
& k_{1}^{*}=1 \\
& I-k_{2}^{*} R_{1}=0 \\
& k_{3}^{*}=\frac{1}{m_{1}}
\end{aligned}
$$

or the following ones:

$$
\begin{aligned}
& \left(4 m_{2}^{2}-4 m_{1} m_{2}\right)\left(k_{2}^{*}\right)^{2}+\left(4 m_{2} m_{3}-4 m_{1} m_{3}\right) k_{2}^{*} \\
& +\left(m_{3}^{2}-4 m_{1}\right)=0 \\
& k_{3}^{*}=\frac{-2 m_{2} k_{2}^{*}+m_{3}}{2 m_{1}}+\frac{1}{m_{1}} \\
& k_{1}^{*}=\frac{m_{2}\left(k_{2}^{*}\right)^{2}+m_{3} k_{2}^{*}+1}{k_{3}^{*} m_{1}-1}+1
\end{aligned}
$$

Proof: Since there is a strict inequality constraint in Problem 2 (namely $k_{1}>1$ ), the Karush-Kuhn-Tucker (KKT) method cannot be employed here (in fact, if this constraint is replaced by $k_{1} \geq 1$ to enable the exploitation of the KKT method, a wrong result may be obtained in which $k_{3}^{*}=-\infty$ ). Therefore, let all different possibilities be investigated below:

- Case 1: The equation $k_{1}^{*}-1=0$ holds. In this case, the underlying optimization problem reduces to finding the infimum of $1+2 k_{2}+k_{3}$ under the constraint $-1-k_{2}^{2} m_{2}-k_{2} m_{3}>0$. Since the resultant objective function has no local minimum as noted above, the optimal solution occurs at some point on the remaining boundary, i.e. $-1-\left(k_{2}^{*}\right)^{2} m_{2}-k_{2}^{*} m_{3}=0$. On the other hand, it follows from (27) that:

$$
\begin{aligned}
0 & \leq \bar{\lambda}\left(\left(I-k_{2}^{*} R_{1}\right)\left(I-k_{2}^{*} R_{1}^{T}\right)\right) \\
& \leq 1+k_{2}^{*} m_{3}+\left(k_{2}^{*}\right)^{2} m_{2}
\end{aligned}
$$

The above relation along with the equation $-1-$ $\left(k_{2}^{*}\right)^{2} m_{2}-k_{2}^{*} m_{3}=0$ signifies that the matrix $I-k_{2}^{*} R_{1}$ is equal to zero. Taking this result into account, it can be concluded from the constraint of Problem 2 and the equation $k_{1}^{*}=1^{+}$that $k_{3}^{*} m_{1}-1$ is nonnegative. This implies that in order for the objective function to be minimized, $k_{3}^{*}$ should be chosen as $\frac{1}{m_{1}}$. The relations obtained above satisfy the set of equations given in (28).

- Case 2: The equation $\left(k_{1}^{*}-1\right)\left(-1+k_{3}^{*} m_{1}\right)-1$ $\left(k_{2}^{*}\right)^{2} m_{2}-k_{2}^{*} m_{3}=0$ holds. It can be inferred in this case that:

$$
k_{1}^{*}=\frac{m_{2}\left(k_{2}^{*}\right)^{2}+m_{3} k_{2}^{*}+1}{k_{3}^{*} m_{1}-1}+1
$$

If $k_{1}^{*}-1$ is equal to zero, this case turns out to be the same as case 1 . Hence, with no loss of generality, 
assume that $k_{1}^{*}-1$ is strictly positive. This yields that solving Problem 2 is equivalent to finding the lowest minimum point of the function:

$$
\frac{m_{2} k_{2}^{2}+m_{3} k_{2}+1}{k_{3} m_{1}-1}+1+2 k_{2}+k_{3}
$$

for which $k_{1}^{*}$ obtained in (31) is greater than or equal to 1 . Taking the gradient of the above function and equating it to zero will lead to the equations:

$$
\begin{aligned}
& \frac{m_{2}\left(k_{2}^{*}\right)^{2}+m_{3} k_{2}^{*}+1}{\left(k_{3}^{*} m_{1}-1\right)^{2}} \times\left(-m_{1}\right)+1=0 \\
& 2+\frac{2 m_{2} k_{2}^{*}+m_{3}}{m_{1} k_{3}^{*}-1}=0
\end{aligned}
$$

One can combine these two equations to arrive at the relation $(29 \mathrm{a})$. The proof follows from the fact that the equations (33b) and (31) are identical to (29b) and $(29 \mathrm{c})$, respectively.

- Case 3: The strict inequalities $\left(k_{1}^{*}-1\right)\left(-1+k_{3}^{*} m_{1}\right)-$ $1-\left(k_{2}^{*}\right)^{2} m_{2}-k_{2}^{*} m_{3}>0$ and $K_{1}^{*}>1$ both hold. As shown in case 1 , the relation $k_{3}^{*} m_{1}-1 \geq 0$ must hold. This implies that there exists a sufficiently small positive number $\eta$ such that the triple $\left(k_{1}^{*}-\eta, k_{2}^{*}, k_{3}^{*}\right)$ satisfies the constraints of Problem 2, while the value of the objective function at this triple is smaller than its minimum value. This contradiction rules out this case.

Theorem 3 presents a solution to Problem 2, which in turn (according to Theorem 2) provides a value for $\mu$ (i.e., the suboptimality degree of the controller $\mathcal{K}$ ). Regarding the set of equations (29) in this theorem, one should note that the quadratic equation (29a) needs to be solved first. The solution should then be substituted into the equations (29b) and (29c) to find all other parameters.

The question arises, how conservative are the values of $\mu$ obtained in Theorems 1 and 2? To answer this question, an elegant result on the tightness of this bound will be presented next.

Theorem 4 In the case where $A_{h}$ and $A_{c}$ are identical, Theorems 1 and 2 both arrive at the exact solution $\mu=0$.

Proof: If $A_{h}=A_{c}$, then it can be easily verified that $R_{1}=R_{2}=-I$, and consequently $m_{1}=m_{2}=1$ and $m_{3}=2$. Now, Theorem 2 states (after some simplifications) that $\mu$ is equal to the infimum of $k_{1}+2 k_{2}+k_{3}$ under the inequality constraints $k_{1}>1$ and:

$$
k_{1} k_{3}-k_{1}-k_{3}-2 k_{2}-k_{2}^{2}>0
$$

The latter inequality is equivalent to:

$$
\left(k_{1}-1\right)\left(k_{3}-1\right)>\left(k_{2}+1\right)^{2}
$$

Hence, $\mu$ is equal to 0 , and is attained when $k_{1}=k_{3} \rightarrow$ $1^{+}$and $k_{2}=-1$.

Remark 5 It can be inferred from Theorem 4 and the continuity, that if $A_{c}$ is sufficiently close to $A_{h}$, then the upper bounds proposed in this paper will be arbitrarily close to zero. As can be noticed from the proof of this theorem, the result is not trivial at all. In other words, it is not straightforward to conclude from Theorems 1 and 2 that if $A_{c}=A_{h}$, then the corresponding upper bounds will be equal to the exact value, implying $\mu=0$.

Remark 6 The results obtained in this work can be analogously developed to tackle the following problem:

Assume that the system $\mathcal{S}$ (which is not necessarily hierarchical) is subject to perturbation. Design a LTI decentralized controller for the nominal model of the system. Now, the matrices $A_{h}$ and $A_{c}$ correspond to the closedloop nominal and the closed-loop perturbed $A$-matrices in the state space representation, respectively. In this case, the ratio $\frac{J_{d}}{J_{c}}$ describes the closeness of the nominal closedloop system and its perturbed counterpart. As in the previous problem, this ratio would be very useful in evaluating the performance of a physical system under a controller designed for the nominal model of the system.

\section{Numerical Example}

Example 1: Consider an overlapping system $\mathcal{S}$ given by:

$$
x[k+1]=A x[k]+B u[k]
$$

where:

$$
A=\left[\begin{array}{cc|c}
1 & 0 & 0 \\
\cline { 2 - 3 } 0.1 & 0.3 & -0.1 \\
\hline 0 & 1 & 0
\end{array}\right], \quad B=\left[\begin{array}{c|c}
0.5 & 0 \\
0 & 0 \\
\hline 0 & 0.5
\end{array}\right]
$$

with the overlapping part $A_{22}$. The objective is to design an overlapping controller for this system with the control law $u[k]=\mathcal{K} x[k]$, which minimizes the performance index $J=\sum_{0}^{\infty} x[k]^{T} x[k]+u[k]^{T} u[k]$. Notice that the matrix $\mathcal{K}$ to be designed must have the following structure:

$$
\mathcal{K}=\left[\begin{array}{lll}
* & * & 0 \\
0 & * & *
\end{array}\right]
$$

A prevailing approach to achieve this objective is to exploit the expansion-contraction method (Chu et al., 
2005). Using a transformation matrix $V$ given by:

$$
V=\left[\begin{array}{lll}
1 & 0 & 0 \\
0 & 1 & 0 \\
0 & 1 & 0 \\
0 & 0 & 1
\end{array}\right]
$$

an expanded system $\mathcal{S}_{e}$ can be obtained as follows (Bakule et al., 2004, 2001):

$$
x_{e}[k+1]=A_{e} x_{e}[k]+B_{e} u_{e}[k]
$$

where:

$$
A_{e}=\left[\begin{array}{cccc}
1 & 0 & 0 & 0 \\
0.1 & 0.3 & 0 & -0.1 \\
0.1 & 0 & 0.3 & -0.1 \\
0 & 0 & 1 & 0
\end{array}\right], \quad B_{e}=\left[\begin{array}{cc}
0.5 & 0 \\
0 & 0 \\
0 & 0 \\
0 & 0.5
\end{array}\right]
$$

A controller $u_{e}[k]=\mathcal{K}_{e} x_{e}[k]$ is now to be designed for the system $\mathcal{S}_{e}$ to minimize the performance index $J_{e}=\sum_{0}^{\infty} x_{e}[k]^{T} x_{e}[k]+u_{e}[k]^{T} u_{e}[k]$, where, according to the overlapping technique, the matrix $\mathcal{K}_{e}$ must have the following form:

$$
\mathcal{K}_{e}=\left[\begin{array}{cccc}
* & * & 0 & 0 \\
0 & 0 & * & *
\end{array}\right]
$$

To simplify the control design problem, one can use the overlapping technique to replace the matrix $A_{e}$ with the following:

$$
\bar{A}_{e}=\left[\begin{array}{cccc}
1 & 0 & 0 & 0 \\
0.1 & 0.3 & 0 & 0 \\
0 & 0 & 0.3 & -0.1 \\
0 & 0 & 1 & 0
\end{array}\right]
$$

Consequently, the optimal decentralized controller for the expanded system $\mathcal{S}_{e}$ (using the above matrix) is given by:

$$
\mathcal{K}_{e}=\left[\begin{array}{cccc}
-0.7867 & -0.0037 & 0 & 0 \\
0 & 0 & -0.3997 & -0.0022
\end{array}\right]
$$

It is worth mentioning that since $\bar{A}_{e}$ is a block-diagonal matrix, it represents two decoupled subsystems, and hence designing $\mathcal{K}_{e}$ is reduced to finding two separate controllers, which is easier in general. The correspond- ing near-optimal controller for the original system $\mathcal{S}$ is:

$$
\mathcal{K}=\left[\begin{array}{ccc}
-0.7867 & -0.0037 & 0 \\
0 & -0.3997 & -0.0022
\end{array}\right]
$$

Since the matrix $\mathcal{K}_{e}$ is designed for the system $\mathcal{S}_{e}$ with the parameters $\left(\bar{A}_{e}, B_{e}\right)$ instead of $\left(A_{e}, B_{e}\right)$, it is desired to verify how this would affect the performance of the closed-loop system. For this purpose, consider the matrices $A_{h}$ and $A_{n h}$ as $\bar{A}_{e}+B_{e} \mathcal{K}_{e}$ and $A_{e}+B_{e} \mathcal{K}_{e}$, respectively. Using Theorem 1 , one can easily obtain $k_{1}=1.1909, k_{2}=-1.1955$ and $k_{3}=1.2240$, which leads to a suboptimal controller with the performance index 0.024. The smallness of this number confirms that neglecting the interconnections in the proposed design procedure does not result in a noticeable performance degradation. It is to be noted that the exact value of $\frac{J_{d}}{J_{h}}$ in this example is equal to 0.0116 . The result of this example is in accordance with the discussion in Remark 1.

Example 2: Consider an interconnected system $\mathcal{S}$ with nine SISO subsystems of order 1 , and assume that the interconnections from subsystem $i$ to subsystem $j$, for $i, j \in\{1,2, \ldots, 9\}, i<j$, are in general "weaker" than the ones in the opposite direction. Hence, to design a decentralized controller for the system with nine local controllers, one can eliminate these weak interconnections and design a decentralized controller for the obtained hierarchical model using any existing method. For simplicity, assume that a static decentralized controller has been designed for the hierarchical model. To carry out performance analysis for the pseudo-hierarchical system under the designed controller, two different choices will be considered for the closed-loop matrix $A_{n h}$ in the sequel.

Consider first a matrix $A_{n h}$ of the following form:

$$
\frac{1}{4.35}\left[\begin{array}{ccccccccc}
1 & 0.5 & 2 & 0.1 & 0.5 & 0.6 & 0.3 & 0.3 & 0.1 \\
0 & 1 & 1.5 & 0.5 & 1 & 0 & 1 & 0.2 & 0.25 \\
1 & 0.3 & 1 & 0 & 0.2 & 1 & 0.2 & 0.5 & 0.31 \\
0 & 0 & 0.3 & 1 & 3 & 1 & 0.05 & 0.1 & 0.01 \\
0.3 & 0 & 0 & 1 & 1 & 2 & 0 & 0 & 0.2 \\
0 & 0 & 0 & 0 & 1 & 1 & 0.8 & 0 & 1 \\
0 & 0 & 0.04 & 0.5 & 0.6 & 0 & 0.5 & 1 & 1 \\
0.01 & 0 & 0 & 0.1 & 0.1 & 0 & 0.5 & 1 & 2 \\
0.4 & 0.9 & 0.04 & 0.03 & 0 & 0.3 & 0.05 & 1 & 0
\end{array}\right]
$$

It can be observed that the lower-diagonal entries of this matrix have smaller magnitudes compared to the upperdiagonal ones in general (which translate to "weak" interconnections in the digraph of the system). The hierarchical matrix $\left(A_{h}\right)$ obtained by neglecting the lowerdiagonal entries of the above matrix is given by: 


$$
\frac{1}{4.35}\left[\begin{array}{ccccccccc}
1 & 0.5 & 2 & 0.1 & 0.5 & 0.6 & 0.3 & 0.3 & 0.1 \\
0 & 1 & 1.5 & 0.5 & 1 & 0 & 1 & 0.2 & 0.25 \\
0 & 0 & 1 & 0 & 0.2 & 1 & 0.2 & 0.5 & 0.31 \\
0 & 0 & 0 & 1 & 3 & 1 & 0.05 & 0.1 & 0.01 \\
0 & 0 & 0 & 0 & 1 & 2 & 0 & 0 & 0.2 \\
0 & 0 & 0 & 0 & 0 & 1 & 0.8 & 0 & 1 \\
0 & 0 & 0 & 0 & 0 & 0 & 0.5 & 1 & 1 \\
0 & 0 & 0 & 0 & 0 & 0 & 0 & 1 & 2 \\
0 & 0 & 0 & 0 & 0 & 0 & 0 & 0 & 0
\end{array}\right]
$$

In can be verified that $J_{d}$ and $J_{h}$ for this example are equal to 67.9336 and 38.6145 , respectively, and hence $\frac{J_{d}}{J_{h}}=1.7593$. On the other hand, an upper bound on $\frac{J_{d}}{J_{h}}$ can be obtained from Theorem 1 by solving Problem 1 , which results in:

$$
k_{1}=2.131, \quad k_{2}=-1.7529, \quad k_{3}=5.7894
$$

From the relation $\mu=\min \left(k_{1}+2 k_{2}+k_{3}\right)$, the upper bound $\mu$ on the ratio $\frac{J_{d}}{J_{h}}$ is equal to 4.4145 . Note that although the resultant upper bound is approximately 2.5 times greater than the exact value, it is attained through a quite simple procedure, which is very desirable for large-scale systems. This relatively large difference between $\frac{J_{d}}{J_{h}}$ and the corresponding upper bound $\mu$ is due to the fact that the neglected interconnections are not "weak" enough to be ignored. For instance, there are some large lower-triangular entries (such as $\frac{0.9}{4.35}$ ), which are comparable to and even greater than some of the upper-triangular entries.

Since Problem 1 involves matrix variables, it may not be easily handled for large-scale systems. Thus, let Theorems 2 and 3 be utilized here to find an upper bound on $J_{d} / J_{h}$. In this case, $k_{1}, k_{2}$ and $k_{3}$ are equal to 1.5206 , -0.9144 and 5.2813, respectively, which lead to the upper bound $\mu=4.973$. Although the matrix constraint in Problem 1 may seem to be oversimplified in obtaining Problem 2, the results of Example 2 show that this is not necessarily the case. In other words, the bound $\mu=4.973$ obtained by using Theorem 3 is relatively close to the bound $\mu=4.4145$ resulted from Theorem 1 .

Example 3: Consider the matrix $A_{n h}$ as follows:

$$
\frac{1}{4.35}\left[\begin{array}{ccccccccc}
1 & 0.5 & 2 & 0.1 & 0.5 & 0.6 & 0.3 & 0.3 & 0.1 \\
0 & 1 & 1.5 & 0.5 & 1 & 0 & 1 & 0.2 & 0.25 \\
0 & 0 & 1 & 0 & 0.2 & 1 & 0.2 & 0.5 & 0.31 \\
0 & 0 & 0 & 1 & 3 & 1 & 0.05 & 0.1 & 0.01 \\
0 & 0 & 0 & 0 & 1 & 2 & 0 & 0 & 0.2 \\
0.05 & 0 & 0 & 0 & 0 & 1 & 0.8 & 0 & 1 \\
0 & 0 & 0 & 0 & 0 & 0 & 0.5 & 1 & 1 \\
0 & 0 & 0 & 0 & 0 & 0 & 0 & 1 & 2 \\
0.001 & 0 & 0 & 0 & 0 & 0 & 0 & 0 & 0
\end{array}\right]
$$

Here, the "weak" interconnections of the previous system have been further weakened in order for the pseudohierarchical system to become closer to its reference hierarchical model. Notice that the reference hierarchical model of this system is the same as the one in the previous example, i.e. (46). It can be verified that $J_{d}$ and $J_{h}$ in this case are equal to 0.0117 and 38.6145 , respectively. The substantial drop in the magnitude of $J_{d}$ compared to the previous case, manifestly confirms that the closeness of the pseudo-hierarchical and the corresponding reference hierarchical models would have a significant impact on the effectiveness of the proposed performance evaluation. The upper bound $\mu$ obtained by using Theorems 1 and 2 are equal to 0.0041 and 0.0671 , respectively.

\section{Conclusions}

This paper deals with the performance analysis of largescale systems with pseudo-hierarchical structures; i.e., those systems whose transfer function matrix is "close" to being hierarchical, due to some weak elements (interconnections) in it. It is assumed that a stabilizing decentralized controller is available for the system. This controller is basically designed for a reference hierarchical model; i.e., a hierarchical model which is obtained by eliminating some weak interconnections in the original system. The controller obtained by using this indirect design technique may not perform well when applied to the original pseudo-hierarchical system. A proper LQ cost function is defined to measure the discrepancy between the performance of the original system and its hierarchical counterpart, under the above controller. Since computing the exact value of this cost function involves a large-scale Lyapunov equation, in practice it is preferable to obtain an upper bound on it. Hence, a simple LMI optimization problem with only three variables is proposed to attain this upper bound. To further simplify the procedure, the matrix optimization problem is reduced to a scalar one for which an explicit solution is obtained. In addition, it is shown that the closer the pseudo-hierarchical system to the reference hierarchical model is, the smaller these bounds are. In the special case when the two models are identical, these bounds are 
both equal to zero. This demonstrates that the bounds obtained by using the simplified optimization problems are not too conservative. The usefulness of the proposed techniques is illustrated by three numerical examples.

\section{References}

A. C. Antoulas and D.C. Sorensen, "Approximation of large-scale dynamical systems: An overview," International Journal of Applied Mathematics and Computer Science, vol. 11, no. 5, pp. 1093-1121, 2001.

M. Jamshidi, "Large-scale systems: modeling, control, and fuzzy logic," Prentice-Hall, NJ, 1997.

J. Lavaei, A. Momeni and A. G. Aghdam, "Highperformance decentralized control for formation flying with leader-follower structure," in Proc. 45th IEEE Conference on Decision and Control, San Diego, CA, pp. 5947-5954, 2006.

J. Lavaei and A. G. Aghdam, "High-performance decentralized control design for general interconnected systems with applications in cooperative control," International Journal of Control, vol. 80, no.6, pp. 935-951. 2007.

J. Lavaei, A. Momeni and A. G. Aghdam, "A Model Predictive Decentralized Control Scheme with Reduced Communication Requirement for Spacecraft Formation," IEEE Transactions on Control Systems Technology, vol. 16, no. 2, pp. 268-278, 2008.

H. G. Tanner, G. J. Pappas and V. Kumar, "Leader to formation stability," IEEE Transactions on Robotics and Automation, vol. 20, no. 3, pp. 443455, 2004.

J. A. Fax and R. M. Murray, "Information flow and cooperative control of vehicle formations," IEEE Transactions on Automatic Control, vol. 49, no. 9, pp. 1465-1476, 2004.

D. J. Stilwell and B. E. Bishop, "Platoons of underwater vehicles," IEEE Control Systems Magazine, vol. 20, no. 6, pp. 45-52, 2000.

A. G. Aghdam, E. J. Davison and R. Becerril, "Structural modification of systems using discretization and generalized sampled-data hold functions," $A u$ tomatica, vol. 42, no. 11, pp. 1935-1941, 2006.

R. Becerril, A. G. Aghdam and E. J. Davison, "Minimizing interconnection of subsystems in large-scale interconnected systems using generalized sampling," in Proc. 16th IFAC World Congress, Prague, Czech Republic, 2005.

A. Iftar, "Overlapping decentralized dynamic optimal control," International Journal of Control, vol. 58, no. 1, pp. 187-209, 1993.

S. S. Stankovic and D. D. Šiljak, "Sequential LQG optimization of hierarchically structured systems," $A u$ tomatica, vol. 25, no. 4, pp. 545-559, 1989.

D. D. Šiljak, "Decentralized control of complex systems," Boston: Academic Press, 1991.

S. S. Stankovic, M. J. Stanojevic, and D. D. Siljak, "Decentralized overlapping control of a platoon of vehi- cles," IEEE Transactions on Control Systems Technology, vol. 8, no. 5, pp. 816-832, 2000.

D. D. Šiljak and A. I. Zecevic, "Control of large-scale systems: Beyond decentralized feedback," Annual Reviews in Control, vol. 29, no. 2, pp. 169-179, 2005.

A. I. Zecevic and D. D. Šiljak, "A new approach to control design with overlapping information structure constraints," Automatica, vol. 41, no. 2, pp. 265272, 2005.

D. Chu and D. D. Šiljak, "A canonical form for the inclusion principle of dynamic systems," SIAM Journal on Control and Optimization, vol. 44, no. 3, pp. 969-990, 2005.

R. Krtolica and D. D. Šiljak, "Suboptimality of decentralized stochastic control and estimation," IEEE Transactions on Automatic Control, vol. 25, no. 1, pp. 76-83, 1980.

C. H. Lee, "Solution bounds of the continuous and discrete Lyapunov matrix equations," Journal of $\mathrm{Op}$ timization Theory and Applications, vol. 120, no. 3, pp. 559-578, 2004.

L. Bakule, J. Rodellar and J. M. Rossell, "Inclusion principle for uncertain discrete-time systems with guaranteed cost," in proceedings of 43rd IEEE Conference on Decision and Control, Atlantis, Paradise Island, Bahamas, pp. 2712-2716, 2004.

L. Bakule, J. Rodellar, J. M. Rossell and P. Rubió, "Preservation of controllability-observability in expanded systems," IEEE Transactions on Automatic Control, vol. 46, no. 7, pp. 1155-1162, 2001.

M. E. Sezer and D. D. Siljak "Nested $\varepsilon$-decompositions and clustering of complex systems," Automatica, vol. 22, no. 3, pp. 321-331, 1986.

M. E. Sezer and D. D. Siljak "Nested epsilon decompositions of linear systems: Weakly coupled and overlapping blocks," SIAM Journal of Matrix Analysis and Applications, vol. 12, pp. 521-533, 1991.

A. I. Zecevic and D. D. Šiljak "A block-parallel Newton method via overlapping epsilon decompositions," SIAM Journal of Matrix Analysis and Applications, vol. 15, pp. 824-844, 1994.

J. Löfberg, "A toolbox for modeling and optimization in MATLAB," in Proceedings of the CACSD Conference, Taipei, Taiwan, 2004 (available online at http://control.ee.ethz.ch/ joloef/yalmip.php).

S. Prajna, A. Papachristodoulou, P. Seiler and P. A. Parrilo, "SOSTOOLS sum of squares optimization toolbox for MATLAB," Users guide, 2004 (available online at http://www.cds.caltech.edu/sostools).

L. N. Trefethen and D. Bau, "Numerical Linear Algebra," SIAM, 1997.

M. K. Tippett and D. Marchesin, "Upper bounds for the solution of the discrete algebraic Lyapunov equation," Automatica, vol. 35, no. 8, pp. 1485-1489, 1999.

P. Benner, "Large-scale matrix equations of special type," Numerical Linear Algebra with Applications, vol. 15, no. 9, pp. 747-754, 2008. 


\section{APPENDIX}

It is desired to show that since the matrix $P_{h}$ is the solution of a Lyapunov equation involving a hierarchical matrix $\left(A_{h}\right)$, it can alternatively be found by solving a number of Lyapunov and Sylvester equations of subsystems' orders (as opposed to the system's order), successively. To streamline the argument, assume that there are only two subsystems (i.e. $\nu=2$ ). In this case, the equation $A_{h}^{T} P_{h} A_{h}-P_{h}+I=0$ can be equivalently decomposed as:

$$
\begin{aligned}
& A_{22}^{T} P_{3} A_{22}-P_{3}+I=0 \\
& A_{11}^{T} P_{2} A_{22}+A_{21}^{T} P_{3} A_{22}-P_{2}=0 \\
& A_{11}^{T} P_{1} A_{11}+A_{11}^{T} P_{2} A_{21}+A_{21}^{T} P_{2} A_{11} \\
& +A_{21}^{T} P_{3} A_{21}-P_{1}+I=0
\end{aligned}
$$

where:

$$
P_{h}=\left[\begin{array}{ll}
P_{1} & P_{2} \\
P_{2} & P_{3}
\end{array}\right]
$$

Since the hierarchical closed-loop system is stable, the matrices $A_{11}$ and $A_{22}$ are both Schur. Thus, the Lyapunov equation (.1a) which is of subsystem's order, can be solved to find the matrix $P_{3}$. Substitution of $P_{3}$ in the equation (.1b) will yield a Sylvester equation, which has a unique solution $P_{2}$ (because the eigenvalues of $A_{11}$ and $A_{22}$ are all inside the unit circle). Finally, the Lyapunov equation (.1c) can be solved for the matrix variable $P_{1}$ after substituting $P_{2}$ and $P_{3}$ obtained above into this equation. This illustrates that due to the special structure of $A_{h}$, Assumption 1 is not essential for hierarchical systems. An analogous method can be adopted in the general case (i.e. $\nu>2$ ), in order to obtain the Lyapunov matrix corresponding to the large-scale system through a series of Sylvester and Lyapunov equations of smaller sizes.

One may think that the above-mentioned back substitution method is potentially problematic, because solving a hierarchy of equations could lead to the propagation of the round-off error and eventually a numerical instability. However, the Gaussian elimination method for solving a linear upper-triangular system (which is a certain type of back substitution technique), is known to be numerically stable (Trefethen et al., 1997). It can be similarly shown that the aforementioned method is numerically efficient too. To clarify this, let the $(i, j)$ block entry of $P_{h}$ be denoted by $P_{i j}$, and assume that solving the Sylvester equation associated with the variable $P_{i j}$, $i, j \in \bar{\nu}, i \leq j$, yields the solution $\tilde{P}_{i j}$, which is subject to round-off error. It can be verified that:

$$
A_{h}^{T} \tilde{P} A_{h}-\tilde{P}=-I+A_{d}^{T} \Delta A_{d}-\Delta
$$

where:
- $\tilde{P}$ is a matrix whose $(i, j)$ block entry is $\tilde{P}_{i j}$, for all $i, j \in \bar{\nu}$.

- $A_{d}$ is a block diagonal matrix with the $(i, i)$ block entry $A_{i i}$, for all $i \in \bar{\nu}$.

- $\Delta$ is a matrix with the $(i, j)$ block entry $\Delta_{i j}$, for all $i, j \in \bar{\nu}$, where $\Delta_{i j}$ is a matrix of the same dimension as $P_{i j}$, representing the round-off error produced in the course of solving the Sylvester equation associated with $P_{i j}$.

The equation (.3) somehow indicates that there does not exist any error propagation phenomenon, and as long as $\Delta$ is small, $\tilde{P}$ would be close to $P_{h}$. More precisely, one can write:

$$
A_{h}^{T}\left(\tilde{P}-P_{h}\right) A_{h}-\left(\tilde{P}-P_{h}\right)=A_{d}^{T} \Delta A_{d}-\Delta
$$

Since all entries of $\Delta$ are small (say, of order $O(\varepsilon)$ ) and $A_{d}$ is block diagonal, one would expect to obtain a solution $\tilde{P}-P_{h}$ of small norm (see Tippett et al. (1999) and references therein for relating the norm of $\tilde{P}-P_{h}$ to the norm of $\Delta$ ). This confirms that the proposed method is not prone to the propagation error, i.e. $\tilde{P}$ is normally close to $P_{h}$.

The other issue about the proposed back substitution method is that it significantly reduces the computational burden, provided the orders of the subsystems are sufficiently smaller than the order of the system. For instance, assume that all subsystems have roughly the same dimension, say $\frac{n}{\nu}\left(n:=n_{1}+\cdots+n_{\nu}\right)$. Then, the computational complexity of the Lyapunov solution $P_{h}$ using the existing efficient methods is $O\left(n^{3}\right)$ (Benner, 2008). In contrast, by taking the particular structure of $A_{h}$ into account, the present method requires that $\frac{\nu(\nu+1)}{2}$ Sylvester equations be solved, whose overall complexity order is $\nu^{2} \times O\left(\left(\frac{n}{\nu}\right)^{3}\right)$. This implies that the proposed algorithm reduces the computational complexity by a factor of $\frac{1}{\nu}$. 